\title{
RELAPSE OF THE BUG AT A DIFFERENT SITE- A CASE OF MULTIDRUG RESISTANT MELIOIDOSIS
}

\author{
Chitralekha Anilkumar Nayak1, Nicasia Furtado², Edwin Gomes³, Shruthi Kulkarni
}

${ }_{1}^{1}$ Senior Resident, Department of Medicine, Goa Medical College, Bambolim, Goa. ${ }^{2}$ Assistant Professor, Department of Medicine, Goa Medical College, Bambolim, Goa.

3Professor, Department of Medicine, Goa Medical College, Bambolim, Goa.

4Junior Resident, Department of Medicine, Goa Medical College, Bambolim, Goa.

HOW TO CITE THIS ARTICLE: Nayak CA, Furtado N, Gomes E, et al. Relapse of the bug at a different site- a case of multidrug resistant melioidosis. J. Evolution Med. Dent. Sci. 2018;7(17):2163-2164, DOI: 10.14260/jemds/2018/484

\section{PRESENTATION OF CASE}

A 60-year-old farmer who is diabetic for five years presented with pyrexia of unknown origin for one month. Fever was high grade associated with chills, dysuria and increased frequency of micturition, dull aching diffuse abdominal pain and weight loss. There was no history of rash, vomiting, altered bowel habits, cough, bleeding manifestations, altered sensorium and seizures. Patient denied history of recent travel, consumption of raw milk and contact with tuberculosis.

Melioidosis, an emerging underdiagnosed infectious disease is caused by Burkholderia pseudomallei, a Gram negative bacillus commonly found in the soil of tropical countries. It is important to consider melioidosis as a differential diagnosis amongst pyrexia of unknown origin cases even in non-endemic areas for an early antibiotic treatment and prevention of relapse.[1] Almost all B. pseudomallei strains are susceptible to the first line antibiotic Ceftazidime. We report a case of culture proven melioidosis primarily resistant to ceftazidime who developed relapse at different sites in the eradication phase of treatment despite adequate course of in vitro sensitive antibiotics.

\section{DIFFERENTIAL DIAGNOSES}

- Complicated urinary tract infection.

- Tuberculosis.

- Abdominal abscess.

- Occult abdominal malignancy.

\section{CLINICAL DIAGNOSIS}

On examination, patient's vital parameters were stable. There was pallor, icterus, diffuse abdominal tenderness and mild hepatosplenomegaly. The examination of other systems was unremarkable. Laboratory investigations revealed anaemia, leukocytosis with total counts of $20000 / \mathrm{mm}^{3}$ with neutrophilic predominance (Haemoglobin- $9.3 \mathrm{gm} \%$, neutrophil count- $90 \%$, platelet count- $4 \mathrm{lakh} / \mathrm{mm}^{3}$ ). Serum creatinine was $1.5 \mathrm{mg} / \mathrm{dL}$ and there was also a rise in bilirubin (Total bilirubin $4.4 \mathrm{mg} / \mathrm{dL}$, direct bilirubin $2 \mathrm{mg} / \mathrm{dL}$ ) with normal liver enzymes. Urine routine microscopy revealed 60 to 80 pus cells. Tests for malaria, leptospirosis, enteric fever,

'Financial or Other Competing Interest': None.

Submission 08-03-2018, Peer Review 08-04-2018,

Acceptance 14-04-2018, Published 23-04-2018.

Corresponding Author:

Dr. Chitralekha Anilkumar Nayak,

Rukmini, La Marcela Colony,

Marcela-403107, Goa.

E-mail: nayakchitralekha@gmail.com

DOI: $10.14260 /$ jemds $/ 2018 / 484$

\section{(c) (i) $९$}

urine culture, IgM Brucella, HIV Elisa and Mantoux test were found to be negative. Chest x-ray and echocardiography was normal. Abdominal ultrasound revealed hepatosplenomegaly with internal echoes in the bladder. Patient was empirically started on ceftriaxone in view of urinary tract infection. On the fourth day of admission, patient developed spontaneous thrombophlebitis of the left cephalic vein. Doppler revealed complete thrombosis of left cephalic vein extending into subclavian vein. CT scan of thorax and abdomen revealed multiple small hypodense areas in liver (Figure 1), spleen and prostate (Figure 2) showing peripheral enhancement suggestive of microabscesses and bilateral bulky echogenic kidneys with minimal perinephric fluid. Primary BACTEC blood culture revealed significant growth of Burkholderia pseudomallei.

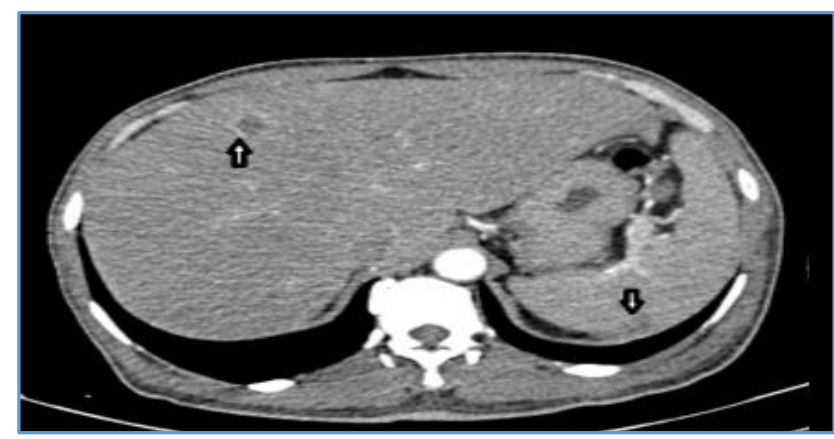

Figure 1. CT Abdomen showing Multiple Hypoechoic Lesions in the Liver and Spleen suggestive of Abscesses

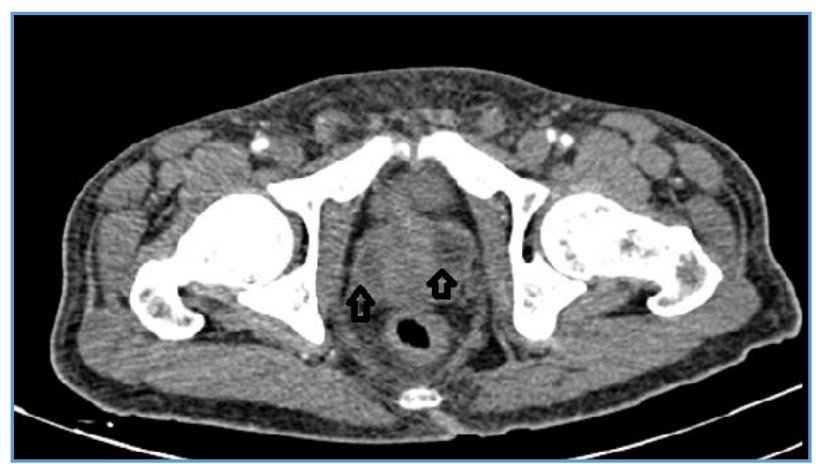

Figure 2. CT Abdomen showing Multiple Hypoechoic Lesions suggestive of Prostatic Abscesses

\section{MANAGEMENT}

Patient was empirically started on ceftazidime, but he did not respond even after eight days of ceftazidime and continued to have high-grade fever with high total leucocyte counts. B. pseudomallei were in vitro strongly sensitive to piperacillin and tazobactam, but resistant to ceftazidime. Forty eight hours after initiation of piperacillin and tazobactam, patient became afebrile and total counts improved to $12000 / \mathrm{mm}^{3}$. 
He received IV antibiotics for four weeks. Oral trimethoprimsulphamethoxazole (TMP-SMX) was prescribed for 12 weeks at discharge. Patient was afebrile with total counts of $6000 / \mathrm{mm}^{3}$ and serum creatinine of $1.4 \mathrm{mg} / \mathrm{dL}$ at the time of discharge with good urine output. Repeat ultrasound abdomen showed resolving microabscesses and blood culture was sterile. Seven weeks after discharge patient developed acute left arm abscess with fever, breathlessness and septic shock in spite of TMP-SMX during the eradication phase. Complete haemogram revealed haemoglobin of $7.3 \mathrm{gm} \%$ with total leucocyte counts of $25000 / \mathrm{mm}^{3}$ and serum creatinine of $2.5 \mathrm{mg} / \mathrm{dL}$. Echocardiography was suggestive of pericardial effusion with partial right ventricular collapse. The left arm abscess was drained and patient was empirically started on meropenem. But he developed cardiorespiratory arrest on the same day and in spite of vigorous resuscitative and ionotropic measures, he could not be revived. Post mortem pericardial fluid was negative for tuberculosis. Blood culture sent at admission revealed significant B. pseudomallei resistant to piperacillin-tazobactam, ceftazidime and meropenem indicating relapse of disseminated melioidosis resistant to multiple antibiotics.

\section{DISCUSSION}

Melioidosis predominantly affects adults with risk factors such as farming, diabetes mellitus and chronic renal and liver disease. [1] It has a tendency for abscess formation in multiple organs such as lung, musculoskeletal system, liver, spleen and prostate. There are also case reports of melioidosis presenting as isolated brain abscess, chest wall abscess and osteomyelitis.[1],[2] Melioidosis with cardiac involvement in the form of pericardial effusion is rare and is often combined with septicaemia, for which the mortality rate is $20 \%$ $60 \% .[2],[3]$ Our patient presented as UTI and left subclavian thrombosis with dissemination to liver, spleen, prostate and relapsed at a different site as arm abscess with pericardial effusion which is a rare clinical phenomenon.

Melioidosis is treated by an intensive two weeks course of ceftazidime or a carbapenem followed by at least 12 weeks of oral trimethoprim-sulphamethoxazole to eradicate the organism and prevent relapse. The median time of resolution of fever is nine days. In patients with abscesses, the resolution can take more than a month.

The studies on recurrent melioidosis have shown it to be either a relapse or a reinfection. A relapse is defined as a new presentation with an acute culture confirmed melioidosis after the resolution of the symptoms and the completion of at least the intensive intravenous phase of the antibiotic therapy for the initial melioidosis presentation. ${ }^{[4]}$ A multifocal infection, disseminated melioidosis during the primary episode and duration and compliance of the therapy have been found to be risk factors for relapse.[5] According to Limmathurotsakul et al, the site of relapse is usually the same as that of the primary episode due to the presence of a quiescent infective nidus and granulomas.[6] Such cases were associated with $4 \%$ antibiotic resistance rates. However, our case proved to be contrary to this theory.

Reports of ceftazidime resistance due to its regular use for melioidosis are increasingly emerging from endemic areas such as Malaysia, Australia, Thailand, Singapore and India.[7],[8] It is becoming an important cause of treatment failure and relapse, probably due to the expression of $\beta$ lactamase and efflux pumps. There are also reports of pan drug-resistant B. pseudomallei from southern Karnataka.[9] The rate of primary resistance to ceftazidime in recurrent melioidosis is higher, but is still uncommon.[10] Hence, it is advisable that empirical ceftazidime still remain an acceptable first choice in melioidosis prior to in vitro antibiotic sensitivity results.

\section{REFERENCES}

[1] Currie BJ, Ward L, Cheng AC. The epidemiology and clinical spectrum of melioidosis: 540 cases from the 20 year Darwin prospective study. PLoS Negl Trop Dis 2010;4(11):e900.

[2] Mathai KRT, Bhat KS, Ashraf M, et al. Melioidosis with a pericardial effusion, which relapsed as a chest wall abscess: a rare presentation. J Clin Diagn Res 2013;7(4):746-8.

[3] Chung $\mathrm{HC}$, Lee CT, Lai $\mathrm{CH}$, et al. Non-septicemic melioidosis presenting as cardiac tamponade. Am J Trop Med Hyg 2008;79(3):455-7.

[4] Limmathurotsakul D, Chaowagul W, Chierakul W, et al. Risk factors for recurrent melioidosis in northeast Thailand. Clin Infect Dis 2006;43(8):979-86.

[5] Limmathurotsakul D, Chaowagul W, Chantratita N, et al. A simple scoring system to differentiate between relapse and re-infection in patients with recurrent melioidosis. PLoS Negl Trop Dis 2008;2(10):e327.

[6] Limmathurotsakul D, Chaowagul W, Day NP, et al. Patterns of organ involvement in recurrent melioidosis. Am J Trop Med Hyg 2009;81(2):335-7.

[7] Pillai MG, Faizal BP, Urs VD. Emerging drug resistance in melioidosis. Ann Trop Med Public Health 2014;7(6):263-5.

[8] Behera B, Prasad Babu TL, Kamalesh A, et al. Ceftazidime resistance in Burkholderia pseudomallei: first report from India. Asian Pac J Trop Med 2012;5(4):329-30.

[9] Mukhopadhyay C, Chawla K, Krishna S, et al. Emergence of Burkholderia pseudomallei and pandrug-resistant non-fermenters from southern Karnataka, India. Trans $\mathrm{R}$ Soc Trop Med Hyg 2008;102(Suppl 1):S12-7.

[10] Wuthiekanun V, Amornchai P, Saiprom N, et al. Survey of antimicrobial resistance in clinical Burkholderia pseudomallei isolates over two decades in Northeast Thailand. Antimicrob Agents Chemother 2011;55(11):5388-91. 